\title{
El profesional de la información ante el reto de los medios de comunicación digitales: el caso de la prensa
}

\author{
María Àngels Jiménez \\ María Eulàlia Fuentes \\ Alfons González
}

Universitat Autònoma de Barcelona

\subsection{Resumen}

Se analiza el nivel de aplicación de los procesos documentales en el diseño de los servicios de valor añadido en los medios de comunicación en Internet a través del caso de la prensa diaria. De acuerdo a ello se establece un conjunto de principios de actuación desde un punto de vista documental orientados tanto a mejorar cualitativamente los SVA como a mostrar las oportunidades profesionales que tienen ante sí los documentalistas en su contribución al diseño de los medios de comunicación digitales.

Palabras clave: Prensa electrónica. Gestión documental. Internet. Servicios de valor añadido.

\subsection{Abstract}

The level of application of the documentary processes in the design of the value added services (VAS) in the mass media in Internet is analysed throughout the case of the daily press. A set of principles of performance from a documentary point of view is established, oriented to improve qualitatively VAS as much as to show the professional opportunities that documentalists have in contributing to the design of digital mass media.

Keywords: Electronic journals. Documentary manegement. Internet. Value added services.

\section{Introducción}

Desde la segunda mitad de la década de los años 90 el número de medios de comunicación en Internet no ha dejado de incrementarse. Primero fue la prensa y más tarde los medios audiovisuales. Las características propias de la Red han pro- 
piciado el surgimiento de nuevos servicios de acceso a la información periodística, tanto corriente como retrospectiva. Todo este universo de opciones informativas ha incorporado una amplia gama de servicios de valor añadido (SVA) que ha enriquecido y modificado la concepción tradicional de los medios de comunicación.

La contribución que a continuación presentamos se enmarca en una línea de investigación iniciada en 1998 en el Àrea de Documentació de la Facultat de Ciències de la Comunicació de la Universitat Autònoma de Barcelona dedicada a analizar la aplicación de las técnicas documentales en el diseño de los servicios de valor añadido de los medios de comunicación en Internet. Con anterioridad a esta breve contribución, esta línea de investigación ha dado otros frutos en forma de artículos para revistas especializadas, comunicaciones a congresos, capítulos en monografías, y, últimamente, una tesis doctoral realizada por Àngels Jiménez.

Desde nuestro punto de vista los SVA son un elemento fundamental en la configuración de la prensa digital en Internet. Por SVA entendemos aquellos servicios que se basan en el uso de las tecnologías y en el grado de interactividad que ofrece la Red para proporcionar al lector utilidades y fuentes de información que no están al alcance de los diarios convencionales por las limitaciones tecnológicas intrínsecas de sus versiones impresas. Los SVA adquieren una especial significación por su múltiple aportación a la prensa electrónica:

- Aumentan el prestigio de la publicación y mejoran la imagen corporativa de la empresa periodística.

- Reafirman la personalidad diferenciada del medio digital.

- Contribuyen a la rentabilidad económica de los rotativos. Fidelizar usuarios a través de una oferta de SVA de calidad influye en los niveles de contratación de publicidad.

- Enriquecen la capacidad informativa y comunicativa de las publicaciones.

- Contribuyen a transformar los diarios en sistemas integrados de información y comunicación.

La tipología de SVA analizados desde una perspectiva documental se agrupan en las siguientes categorías:

- Servicios de comunicación y acceso selectivo a información personalizada.

- Servicios de acceso a información retrospectiva: hemerotecas y archivos digitales.

- Bibliotecas de referencia

- Acceso a recursos de información externa al diario a través de la elaboración de directorios selectivos. 
En los casi cuatro años que desde el Àrea de Documentació llevamos investigando sobre el tema hemos centrado nuestra atención en diversos ámbitos de interés. En un primer momento el objetivo fue conocer cómo la prensa española abordaba el reto de Internet, posteriormente analizamos en profundidad las hemerotecas digitales de los principales diarios nacionales y extranjeros, en una nueva etapa el foco de atención fueron los nuevos servicios y sistemas de acceso a información periodística que Internet ha propiciado, finalmente se ha iniciado una aproximación a la realidad de los SVA en los medios de comunicación audiovisual.

Con este bagaje, el objetivo de la presente contribución es esbozar sucintamente la situación de los SVA de los medios de comunicación españoles, a través del caso de la prensa diaria y desde una perspectiva estrictamente documental. A partir de esa panorámica se proponen unas líneas de actuación que contribuyan a mostrar, de un lado, la importancia que adquieren las técnicas documentales en la configuración de SVA, y del otro, las oportunidades que se abren a los profesionales de la documentación por ser éstos quienes mejor pueden llevar a cabo la aplicación de dichas técnicas y la gestión de los servicios.

\section{La situación actual de los servicios de valor añadido en la prensa española en Internet}

Para llevar a cabo esta descripción sintética se ha optado por aplicar alguno de los criterios de evaluación que se suelen utilizar en el análisis de la situación de las unidades de información como bibliotecas o centros de documentación. A pesar de la distancia que las separa de los servicios de valor añadido de los diarios digitales se pueden establecer ciertos paralelismos. A partir de esta idea se concibe el conjunto de los SVA de un medio como una pequeña unidad de información digital en la que se ofrece una gama cada vez más diversa de servicios de información.

\subsection{Adecuación del personal}

Actualmente se detecta una ausencia generalizada de profesionales formados en Documentación encargados del diseño y mantenimiento de los SVA. Hay muy pocas excepciones: La Vanguardia cuenta con un documentalista dedicado a la localización en Internet de materiales de apoyo al trabajo de los redactores. Marca dispone de un equipo que gestiona Marcamedia, la base de datos que sustenta el servicio de venta de imágenes del rotativo. Esta ausencia se explica en parte por la conjunción de dos factores:

1) Pervive un desconocimiento considerable de la profesión y de su alcance para contribuir a la mejora de los SVA. La buena voluntad y la intuición de otros profesionales suplen la participación de documentalistas.

Scire. 8 : 2 (jul.-dic. 2002) 141-152. 
2) Existe una percepción restrictiva de las funciones y actividades que pueden desarrollar los documentalistas en el seno de un medio digital. De este modo se cree más rentable contar con informáticos y periodistas por considerarlos profesionales más polivalentes.

La ausencia de especialistas formados en el ámbito de la Documentación, junto a la falta de recursos técnicos y económicos son los factores que limitan la configuración y desarrollo de los SVA de los medios digitales españoles.

\subsection{Planificación}

La mayoría de los rotativos que han puesto en marcha algún SVA lo han hecho impelidos por el imperativo de la novedad. Estar presentes en la Red se ha entendido como una exigencia empresarial que obligaba ofrecer algo distinto a la versión impresa del diario. Pero la novedad, el carácter distintivo que han constituido los SVA, no ha sido siempre el resultado de un proceso reflexivo definido por un plan que diese respuesta a cuestiones como: qué proporcionar exactamente, a quién y por qué, y sobre todo, a través de qué medios, tanto tecnológicos, económicos como humanos. La ausencia de un proceso de planificación ha condicionando la calidad de buena parte de los SVA de la prensa digital española y ha sido sustituida por proyectos guiados por la improvisación.

\subsection{Organización y estructuración de los servicios}

A la hora de diseñar una unidad de información clásica, uno de los elementos a considerar es la organización del espacio y la ubicación de los diferentes servicios. Así por ejemplo, en una biblioteca es aconsejable que el servicio de información bibliográfica no se encuentre lejos del fondo documental de referencia, o que el de préstamo esté cerca de los terminales de consulta de los catálogos. De la misma manera, los SVA deberían integrarse de forma racional en la web del diario. Las principales insuficiencias en este sentido se concretan en:

- Ubicación poco visible: Existen SVA a los que no se puede acceder desde la página de inicio del diario.

- Señalización deficiente: Pese a la creciente complejidad que han adquirido las sedes de algunas cabeceras, la inexistencia del mapa de la web del diario dificulta la localización de los servicios.

- Dispersión: En muchos diarios que proporcionan acceso a información retrospectiva a través de diferentes sistemas de consulta, éstos suelen estar aislados entre sí. Otro tanto sucede con los directorios, anuarios, agendas y otras fuentes que podrían constituirse como una pequeña biblioteca de referencia agrupada para facilitar su conocimiento y por tanto su aprovechamiento. 
- Falta de coherencia interna que afecta la estructura del propio SVA: Así, por ejemplo, hay diarios que cuentan con un archivo audiovisual consultable aparentemente a través de un listado alfabético. Sólo después de seleccionar el documento deseado el usuario descubre la existencia de un sistema de búsqueda por palabra clave más rápido y selectivo. En otros diarios sólo se tiene noticia de la existencia de un sistema de búsqueda avanzada después de visualizar los resultados de una búsqueda por el sistema que actúa por defecto.

Estas deficiencias repercuten tanto en el lector (confusión, desorientación, pérdida de tiempo e incluso desconocimiento de los servicios del medio) como en el propio diario: la infrautilización de los SVA anula el valor de los recursos dedicados a su diseño y gestión y devalúa su imagen entre la audiencia.

\subsection{Gestión y tratamiento de la información}

El tratamiento de la información en los SVA de acuerdo a criterios documentales es deficiente en líneas generales.

- Selección: En los servicios donde se lleva a cabo algún tipo de selección documental, prácticamente nunca se definen los criterios aplicados. No es posible conocer cómo se priorizan las noticias que se envían a través de los servicios de alerta, ni de acuerdo a qué criterios se incluyen los recursos en un directorio digital. Estos datos ponen de manifiesto cierta arbitrariedad, cuando no falta de rigor en su concepción.

- Análisis documental: En relación a los procesos de descripción formal y de contenido requeridos para el tratamiento de la información almacenada en hemerotecas, archivos de imágenes, directorios de recursos digitales, etc. los diarios españoles adolecen de un tratamiento riguroso. En los pocos casos en que está presente no se basan en normativas estandarizadas. La indización que se lleva a cabo es un proceso automático. Las hemerotecas indizan el texto completo de la noticia y los sistemas de búsqueda actúan sobre los ficheros inversos. Las fotografías no suelen tratarse de forma independiente, se recuperan a partir de la indización del texto que las acompaña al pie de la imagen o del texto completo de las noticias con las que aparecen publicadas. Todo ello provoca importantes problemas de ruido documental en la recuperación. El tratamiento automatizado de la información y la necesidad de usar el lenguaje natural en la recuperación inciden en este problema. La gestión documental de otros materiales como gráficos interactivos, reportajes especiales o vídeos es prácticamente nula, a excepción de algunos servicios de pago como Marcamedia del deportivo Marca. 
- La clasificación pese a estar presente en diversos servicios no se atiene a normativas estandarizadas. Se aplica en la constitución de los directorios de recursos digitales, en la organización temática de material audiovisual, dosieres especiales o gráficos interactivos que suelen consultarse a través de pequeños sistemas de clasificación creados por el personal del diario y que presentan serias deficiencias: categorías demasiado genéricas o mal delimitadas, en algunos casos arbitrarias, sin apenas información sobre su alcance, con un alto componente de subjetividad y muy poco desarrolladas (no suelen subdividirse o lo hacen tan sólo a un primer nivel).

- La difusión: Las herramientas orientadas a la consulta y explotación de estos servicios suelen presentar interfaces poco versátiles, no son habituales las páginas explicativas de las opciones que el usuario tiene para optimizar su rendimiento y las aplicaciones informáticas que las soportan no son las más idóneas para una gestión documental de la información. Todo ello hace que la riqueza informativa que pudieran proporcionar quede mermada por las insuficiencias en los sistemas de acceso y consulta.

La superación de las actuales insuficiencias de los SVA en relación al tratamiento de la información es una tarea compleja y costosa. Exige un nivel de recursos tanto humanos como técnicos y económicos con los que no todos los diarios cuentan. Tan sólo los pocos servicios concebidos como unidades de negocio son dotados de los recursos necesarios para aportar el nivel de calidad y utilidad que anime a los usuarios a pagar por ellos.

\subsection{Evaluación de los servicios}

Actualmente ningún diario conoce, a través de un estudio directo, el nivel de satisfacción de sus lectores y usuarios con los SVA que proporciona. Recurrir al número de visitas nunca puede ofrecer un dato concluyente en este sentido. Sin una información de este calibre es difícil perfeccionar los SVA y adecuarlos a las necesidades de la audiencia. La ausencia de estudios realizados en esta dirección por los propios diarios, después de casi cinco años de prensa española en la Red es un indicio significativo para valorar la consideración que los propios medios otorgan a sus servicios. Se cree que la gratuidad es por el momento su mayor atractivo. Esta creencia, si se transforma en inercia puede alimentar, en cierto modo, la desatención de un aspecto fundamental: formar al lector en el consumo de servicios de calidad y cultivar una audiencia dispuesta a pagar por ellos.

A modo de conclusión, la situación de los SVA de la prensa española, observada desde un punto de vista documental, presenta una serie de insuficiencias explicables por la conjunción de tres factores básicos: 
- Ausencia de profesionales formados en Documentación que participen activamente en las fases de planificación y diseño de los servicios, lo que conduce también a una gestión inadecuada.

- La incorporación tardía a la Red de algunos diarios, sobre todo de alcance local y regional, ha provocado que sus proyectos estén todavía en una fase de desarrollo incipiente.

- Falta de una conciencia clara por parte de los responsables de los medios de comunicación del papel que pueden desempeñar los SVA en la configuración de una prensa digital con personalidad propia, diferenciada de la impresa y con posibilidades de ser rentable económicamente a medio o largo plazo.

\section{Principios de actuación para garantizar la calidad de los SVA de los diarios digitales}

Estos principios se han concebido a partir de las carencias señaladas. El objetivo no es otro que el de ayudar a la mejora de la calidad de los SVA y por extensión a los propios medios.

3.1. Multidisciplinariedad de los equipos encargados del diseño de los SVA, en los que se debería añadir la figura del documentalista a las del informático y periodista. Esto implica trasladar a la concepción de los equipos de trabajo de las ediciones digitales un vínculo arraigado entre profesionales del periodismo y la documentación en el terreno del funcionamiento de la prensa impresa.

3.2. Planificación. El desarrollo de una oferta de SVA ha de comenzar con una planificación muy precisa. Mediante ésta se han de valorar los requerimientos de los diferentes servicios que se quieran proporcionar y la capacidad del medio para responder de forma continuada. Una falta de previsión en este sentido puede hacer invertir tiempo, esfuerzos y dinero en proyectos que, por ausencia de una planificación previa rigurosa, deberán ser desestimados posteriormente. En el momento de hacer frente a este punto se plantearán aspectos como:

- ¿Qué tipo de SVA se desean proporcionar (acceso a información corriente, retrospectiva, referencial)?

- ¿Cuáles son las exigencies de cada uno de los servicios? Aquí se han de contemplar diversos elementos: el volumen de la información a gestionar, su naturaleza, la frecuencia, etc. No es lo mismo crear una pequeña biblioteca de referencia que mantener la hemeroteca o el archivo fotográfico del diario. En el primer caso puede haber una tarea importante de selección y descripción, pero las exigencias de su mantenimiento no serán equiparables a las de hemerotecas o fototecas donde se deben incorporar constantemente los nuevos documentos que genera la publicación.

Scire. $8: 2$ (jul.-dic. 2002) 141-152. 
- Tipo de gestión que requiere: ¿Se trata de un servicio que puede gestionarse automáticamente o con una mínima intervención humana, como por ejemplo, un servicio de suministro de titulares por e-mail, o bien, necesita un tratamiento intelectual intensivo y realizado por especialistas? En este sentido se deberán prever los recursos humanos, técnicos y económicos: aplicaciones informáticas adecuadas, capacidad del servidor, personal cualificado para llevar a buen término esta tarea. En definitiva, se evalúa el coste que puede representar la creación, gestión y mantenimiento del servicio.

- Posibilidades de comercialización: Si una de las metas de los diarios es comercializar sus servicios, éstos han de incorporar suficiente valor para animar a los lectores a pagar por ellos. Las expectativas del usuario se pueden dirigir hacia el acceso a grandes volúmenes de información, a información especializada seleccionada y analizada, y todo ello acompañado por sistemas de consulta ágiles y potentes. Materializar estas expectativas exige replantear la distribución y asignación de los recursos humanos, técnicos y económicos; por tanto es preciso evaluar previamente las posibilidades de éxito en materia de rentabilidad económica conociendo el número y perfil de la audiencia potencial, frecuencia de uso de los servicios, modalidades de consumo (subscripción, pago por servicio).

- Evaluación: Es conveniente prever mecanismos que permitan evaluar el éxito de los servicios, nivel de uso y grado de satisfacción de los usuarios.

3.3. Utilidad: Los SVA digitales han de ser útiles aportando aquello que el lector difícilmente encontrará en otros soportes o sistemas de información. La utilidad se consigue a través de todos o algunos de los factores siguientes:

- Información relevante de acuerdo al nivel de especialización del medio, o al ámbito geográfico o lingüístico en el que se enmarca.

- Volumen de información: Hay casos en los que la cantidad conduce a la calidad del servicio. ¿Tiene la misma utilidad una hemeroteca que conserva sólo los números de la semana en curso que otra que almacene lo publicado en los últimos seis meses?

- Naturaleza de la información: En el entorno Internet cada vez son menores las limitaciones técnicas para gestionar la información gráfica o audiovisual. El lector percibirá claramente las diferencias entre aquellos medios que ofrezcan la consulta de archivos fotográficos y aquellos donde el acceso a este tipo de material no esté previsto.

- Organización de la información: Sólo es útil aquello que llega a ser usado y para ello debe conocerse dónde está. Los SVA han de ser visibles, localizables con facilidad en la página de inicio o a través del mapa de la sede web. La estructuración interna de cada servicio también es esencial. 
Ofrecer mucha información sin ningún tipo de criterio organizativo puede ser tan inútil como no ofrecerla.

- Sistemas de consulta: Un servicio será más útil cuanto más versátil sea su sistema de consulta y recuperación y más rica sea la oferta de puntos de acceso a la información que contenga. ¿Es lo mismo consultar un directorio de empresas a través de un listado alfabético que hacerlo a través de una pequeña base de datos documental en la que se explote la información de forma selectiva?

- Inmediatez en la respuesta: En los servicios de alerta su utilidad se cifra en la rapidez y precisión de las infomaciones que sumistra al lector.

- Ahorro de tiempo y trabajo: En un contexto de superabundancia informativa uno de los elementos que enriquece la utilidad de un SVA es el acceso a informaciones que se adecúen al perfil del lector. Así, por ejemplo, la efectividad real de un servicio como el envío de titulares por e-mail, concebido para agilizar y facilitar al lector la selección de las informaciones de su interés, se enriquecería si los titulares se acompañasen del lead de la noticia, de este modo el lector podría precisar mejor qué desea leer y ahorrar tiempo.

3.4. Variedad tipológica: La transformación de la prensa diaria en un sistema integrado de información y comunicación pasa por incorporar en su concepción la diversidad de tipologías descritas en este breve estudio. Desde este punto de vista cabe contemplar los nuevos medios como el resultado de la integración de:

- Servicios de alerta y difusión selectiva de la información

- Servicios de acceso a información retrospectiva

- Biblioteca de referencia

- Directorios selectivos de recursos Internet

3.5. Universalidad: Este principio pretende llamar la atención sobre la necesidad de cubrir, a través del diseño de una amplia gama de SVA, las diversas naturalezas de la información (textual, gráfica, audiovisual), así como de todas las secciones y servicios que ofrece la prensa impresa. Una atención parcial que no contemple el principio de universalidad supone una infraexplotación de las ventajas del entorno digital e incide de forma negativa en la calidad de los SVA

3.6. Inteligibilidad: Expresa la necesidad que los SVA se diseñen de forma clara y transparente para el lector. Implica una construcción adecuada y la complementación de los servicios con elementos que los integren oportunamente en el contexto global del medio. El lector ha de poder conocer tanto su existencia, ubicación exacta dentro de la web del diario como sus características, prestaciones y funcionamiento. Una información parcial o incompleta sobre estos aspec- 
tos conduce a la infraexplotación de los servicios por parte del usuario, para evitarlo se hace imprescindible:

- Dar acceso a los SVA desde la página de inicio del medio.

- Informar de su existencia en el mapa del web.

- Utilizar sistemas de señalización claros, con etiquetas visibles e identificativas.

- Incluir descripciones detalladas sobre su funcionamiento con páginas de ayuda o ejemplos explicativos. Así, por ejemplo, en el caso de bases de datos documentales o archivos con información retrospectiva se debería indicar la tipología documental que recoge, criterios de inclusión aplicados, cobertura temática, cronológica, etc., estructura del registro, sistemas de consulta, posibilidades y limitaciones del lenguaje de interrogación, criterios de ordenación y visualización de resultados, etc.

3.7. Rigor: Los SVA se deben diseñar de forma metódica y sistematizada, atendiendo especialmente a dos aspectos:

- Soporte tecnológico: La práctica totalidad de servicios se sustentan en aplicaciones informáticas. Su valoración, selección o diseño debe ser una tarea compartida tanto por técnicos informáticos como por los profesionales de la gestión de la información, quienes previamente habrán definido (planificado) qué necesidades deberá satisfacer el servicio. Es decir, el software no debe imponer un tipo de SVA sino que han de ser los objetivos predefinidos los que perfilarán cómo será la aplicación informática.

- Gestión documental de la información, es decir, la aplicación de los principales procesos de la cadena documental.

- La selección es el proceso más presente en los SVA de las ediciones digitales y como primer eslabón de la cadena condiciona la calidad y resultado final de cada uno. El rigor aplicado en este punto implica trabajar de acuerdo a criterios predefinidos y homogéneos. Establecer una política de selección evita la improvisación y es deseable que el usuario tenga noticia de qué política se ha seguido en la creación de una base de datos, o en un directorio de recursos Internet. En relación a este último ejemplo, existen criterios de aplicación prácticamente estandarizados para valorar recursos digitales y fuentes de referencia.

- El análisis de la información es un proceso esencial y cuando la tipología o naturaleza del servicio aconseje la descripción formal o de contenido de los documentos es recomendable hacerlo de forma sistematizada y uniforme, intentando aplicar normativas estandarizadas, herramientas que los documentalistas usan de forma habitual en su trabajo. Así, por ejemplo, sería preferible que los directorios de recursos 
Internet estuvieran concebidos desde criterios documentales y su indización y clasificación respondiera a lenguajes y pautas normalizadas.

3.8. Recuperabilidad: Este principio está íntimamente ligado a la última fase de la cadena documental: la difusión. Es esencial que los SVA se conciban con el objetivo de proporcionar a sus usuarios las máximas opciones para obtener la información que buscan. Ello quiere decir, por ejemplo, que servicios como directorios o archivos, tanto de información textual como de otro tipo, no deberían limitarse a ofrecer un sistema de consulta basado solamente en la navegación por una clasificación o índice. Esta modalidad de acceso de información debería ser la alternativa a una forma básica y principal como la búsqueda de forma selectiva a través del uso de palabras-clave. De este modo, todo este tipo de SVA habrían de concebirse como bases de datos documentales que realmente enriquecieran las opciones de interrogación y explotación de la información que almacenan.

3.9. Personalización: Uno de los aspectos más valiosos de que disponen los SVA es la capacidad de suministrar información que se adecúe a las preferencias individuales de cada lector. Todo servicio que sea susceptible de adaptarse a un perfil predefinido por el usuario debería diseñarse en esa dirección. Los actuales servicios de suministro de titulares por e-mail ¿por qué han de conformarse con proporcionar un producto estandarizado cuando no existen obstáculos técnicos que impidan dar al lector la posibilidad de determinar qué temas le interesan, de qué secciones o cuándo recibirlas?

3.10. Actualización: Pese a que la actualización de los contenidos es inherente a la práctica periodística, el entorno Internet exige una constante revisión de todos aquellos enlaces que vinculan el diario con fuentes de información externas y uno de los principales SVA que basa su interés en este tipo de fuentes son los diretorios de recursos digitales. Un medio que proporcione un servicio así y cuyos enlaces no sean fiables verá devaluada su imagen ante los usuarios. Existen aplicaciones informáticas destinadas a mantener y corregir los enlaces de este tipo de directorios. El principio de actualización incide también entonces en la conveniencia de renovar las aplicaciones informáticas que soportan o ayudan a mantener los SVA, siempre con la finalidad de ofrecer al público las mejoras derivadas de los avances tecnológicos.

Este conjunto de principios conforman un decálogo que se propone contribuir a la consolidación de los medios digitales en España a través del concurso de las técnicas documentales.

\section{Bibliografía}

Fuentes i Pujol, E.; González, A. (1998). La prensa española en Internet: análisis de los servicios de valor añadido. // Jornadas Españolas de Documentación (6enes:

Scire. 8 : 2 (jul.-dic. 2002) 141-152. 
València: 1998). Valencia: Universitat de València, 1998. 281.292.

Jiménez, A.; González, A.; Fuentes i Pujol, María E. (1999). Gestió documental de la informació en els serveies de valor afegit de la premsa espanyola a Internet. // Jornades Catalanes de Documentació (7es: Barcelona: 1999). Les biblioteques i els centres de documentació al segle XXI: peça clau de la societat de la informació. Barcelona: Col-legi Oficial de Bibliotecaris-Documentalistes de Catalunya, 1999. 405-417.

Jiménez, A.; González, A.; Fuentes i Pujol, M.E. (2000). Las hemerotecas digitales de la prensa en Internet. // El Profesional de la Información. 9: 5 (2000) 15-22.

Jiménez, A.; González, A.; Fuentes i Pujol, M.E. (2001). Sistemas y servicios de acceso a información periodística en Internet. // Anuario de Biblioteconomia, Documentación e Información. Bibliodoc. (2000) 85-102. 